\title{
MODELS OF LOW-SPEED FLOW FOR NEAR-CRITICAL FLUIDS WITH GRAVITATIONAL AND CAPILLARY EFFECTS
}

\author{
BY \\ D. L. DENNY (Department of Mathematics, James Madison University, Harrisonburg, VA) \\ AND
}

R. L. PEGO (Dept. of Mathematics and Institute for Physical Science and Technology, University of Maryland, College Park, MD)

\begin{abstract}
We study low-speed flows of a highly compressible, single-phase fluid in the presence of gravity, for example, in a regime appropriate for modeling recent spaceshuttle experiments on fluids near the liquid-vapor critical point. In the equations of motion, we include forces due to capillary stresses that arise from a contribution made by strong density gradients to the free energy. We derive formally simplified sets of equations in a low-speed limit analogous to the zero Mach number limit in combustion theory.

When viscosity is neglected and gravity is weak, the simplified system includes: a hyperbolic equation for velocity, a parabolic equation for temperature, an elliptic equation related to volume expansion, an integro-differential equation for mean pressure, and an algebraic equation (the equation of state). Solutions are determined by initial values for the mean pressure, the temperature field, and the divergence-free part of the velocity field. To model multi-dimensional flows with strong gravity, we offer an alternative to the anelastic approximation, one which admits stratified fluids in thermodynamic equilibrium, as well as gravity waves but not acoustic waves.
\end{abstract}

1. Introduction. Near the liquid-vapor critical point, many of the thermophysical properties of a fluid exhibit a singular behavior. For instance, the isothermal compressibility and the isobaric thermal expansion coefficients, as well as the isobaric specific heat, all diverge strongly at the critical point. Critical enhancement effects are also encountered in the behavior of the thermal conductivity and the viscosity in the vicinity of the critical point, while the thermal diffusivity approaches zero. These singularities play a major role in the thermal equilibration of near-critical fluids.

Understanding the effect of singular fluid properties on dynamics is not always straightforward. For example, it has been shown that even though thermal diffusivity is small,

Received March 3, 1998.

2000 Mathematics Subject Classification. Primary 35M10, 35Q35, 76N10; Secondary 76N15. 
temperature changes in a near-critical fluid can occur rapidly via a mechanism that causes adiabatic pressure changes in the bulk of the fluid [24, 25]. This adiabatic mechanism creates a strong coupling between temperature changes occurring at the fluid boundaries and the temperature response in the interior of the fluid. It works as follows. A temperature perturbation applied at the boundary of a fluid causes an expansion in the fluid near the boundary. Through the medium of sound waves, this produces an adiabatic pressure change in the interior of the fluid, and a consequent change in the temperature, much more rapidly than could be accomplished by thermal diffusion acting alone. Near the critical point, where the fluid becomes highly expandable and compressible, the adiabatic mechanism dominates the early thermal response and creates a "critical speeding-up" phenomenon. This critical speeding-up has been observed in earth-bound and low-gravity experiments $[4,6]$.

In contrast to the short time scale of the thermal response, experimenters have observed a much longer time scale for the equilibration of density variations $[36,15]$. Near the critical point, the divergence of the compressibility and the influence of gravity can create strong macroscopic density gradients (upon which microscopic density fluctuations are superimposed). Although early adiabatic processes act rapidly (within seconds) to accomplish most of the temperature changes, most of the relaxation of the density distribution to a new equilibrium state is a nonadiabatic process driven by the much slower (hours-long) process of heat diffusion.

Recently, Boukari, Pego, and Gammon [5] studied the combined effects of the adiabatic mechanism and earth's gravity on the equilibration process in near-critical xenon, using a system of equations that includes not only the adiabatic effect, but also onedimensional fluid motion and heat advection. In numerical simulations of a temperature step experiment, they found that the onset of a transient diffusive regime occurs about ten times sooner than estimated by Onuki, Hao, and Ferrell [25] in the zero-gravity case, due to the generation of a small temperature gradient by the adiabatic pressure quench in the presence of gravity. Boukari et al. also observed that over periods of many hours, no single, exponentially-decaying mode was ever observed to dominate the diffusive equilibration process. This conclusion is consistent with measurements and computations of Zhong and Meyer [36] and Kogan, Zhong, and Meyer [17].

The conclusions drawn in these works were based on results derived from one-dimensional systems of equations that do not account for multi-dimensional flows. It is not yet clear how multi-dimensional flows affect equilibration under gravity. Zappoli et. al. [35] have performed computations of two-dimensional buoyant flow for a van der Waals fluid fairly near the critical point ( $1 K$ above critical) where stratification effects are not yet very large, using an "acoustic filtering" technique resembling the approach taken in this paper, and have observed an unusual convection pattern. In the zero-gravity situation, R. F. Berg [3] has pointed out certain differences between one-dimensional and corresponding three-dimensional results regarding the late diffusive regime.

The purpose of this paper is to systematically derive systems of multi-dimensional equations that govern the dynamics of a low-speed, highly compressible, single-phase fluid in the presence of gravity. We shall include forces due to capillary stresses that arise from a contribution made to the energy by strong density gradients. Although 
there are no sharp interfaces between phases in equilibrium in the one-phase regime just above the critical temperature, strong gradients can be generated as transients [14]. We anticipate that nonlocal effects related to the energy of "diffuse interfaces" could play a big role in generating transient flows in certain circumstances that are accessible to experimental observation.

Our starting point is the general hydrodynamic equations expressing the conservation of mass, linear momentum, and energy for a compressible fluid with heat conduction and gravity. We suppose that the equation of state is appropriate for conditions near the critical point, and presume that the fluid is in local equilibrium. This implies that the time scale of interest is longer than a local relaxation time and that the critical point is not so near that the correlation length is macroscopic. These assumptions appear to be reasonable for describing the regimes studied in recent experiments.

To account for the influence of steep density gradients on energy, we adopt a constitutive structure compatible with that described in the work of J. E. Dunn and J. Serrin [9]. Dunn and Serrin permit the constitutive quantities (such as the Cauchy stress tensor) to depend upon the gradient of the density as well as upon density and temperature, so that capillary effects can now be included in the equations. We account for such effects in the simplest way, adding a squared gradient term to the Helmholtz free energy density. The resulting system of equations in Sec. 2 can be used to study the influence of hydrodynamics on heat transfer on a time scale appropriate for resolving sound waves.

Here, however, we are interested in relaxation and flow phenomena that occur very slowly compared to the time it takes for sound waves to cross the spatial domain. A systematic procedure for obtaining simplified equations for compressible flows on long time scales was introduced by Rehm and Baum [27] and by Majda and Sethian [21] in the context of combustion theory. In Sec. 3 we apply this procedure to the system at hand. We non-dimensionalize the equations and estimate the size of the various terms, taking parameters appropriate to a typical experiment of interest. To obtain a simplified set of equations, we neglect terms that make the smallest contribution compared to the other terms.

When the effect of gravity is weak, the result is a simplified model in which the leading-order pressure is spatially constant, and which accounts for multi-dimensional fluid motions influenced by the effects of thermal expansion and contraction, gravitational compression, thermal diffusion, viscosity and capillarity. With viscosity included, this simplified model consists of Eqs. (3.12)-(3.16) below, plus the equation of state.

When viscosity is neglected, the simplified system includes a hyperbolic equation (for velocity), a parabolic equation (for temperature), an elliptic equation (related to volume expansion), an ordinary integro-differential equation (for mean pressure), and an algebraic equation (the equation of state). In a forthcoming work [8], we prove that the simplified model equations are evolutionary; i.e., we show that solutions are determined by suitable initial data. The initial data required to determine the solution consist of the temperature field, the mean pressure, and the divergence-free part of the velocity field. The density is determined from the temperature and mean pressure via the equation of state. 
Earth's gravity creates a strongly nonlinear density profile in equilibrium when temperature is close to the critical point, due to the high compressibility. This is not wellmodelled by the system (3.12)-(3.16). In Sec. 4 we study how to model multi-dimensional flows with strong gravity. If we enforce hydrostatic balance at leading order, as was done for the one-dimensional case in [5], we find that generally the flow must remain strictly stratified, and any vertical fluid motions are due only to horizontally uniform density changes.

In order to admit nontrivial convective flows or gravity waves, one can assume that entropy is constant at leading order and neglect heat conduction. This corresponds to the assumptions made by Ogura and Phillips [23] in deriving "anelastic" equations for atmospheric circulations. Here we obtain anelastic equations valid for a general equation of state. The assumption of approximately constant entropy may not be compatible with thermodynamic equilibrium near the critical point, but this assumption may be relevant for describing some experiments that have been performed at near-constant entropy in order to reduce the effect of density gradients $[4,22]$. Because heat conduction is neglected, though, the anelastic equations do not contain the fast adiabatic heat-transfer mechanism mentioned above.

We find a better alternative if we return to the weak-gravity scaling and make a simple modification of the momentum equation. We retain the effect of the pressure correction on density in the gravity force term, in the spirit of the Boussinesq approximation. This modification does not change the formal validity of the approximation. But the new system, consisting of Eqs. (4.18)-(4.20) below, correctly captures strongly stratified thermodynamic equilibria, admits multi-dimensional flow including gravity waves but not acoustic waves, and includes heat conduction and the adiabatic mechanism.

2. Basic hydrodynamic equations. To start, we consider the general hydrodynamic equations expressing the conservation of mass, linear momentum, and energy for a compressible fluid with heat conduction and gravity:

$$
\begin{aligned}
\frac{D \rho}{D t} & =-\rho \nabla \cdot \mathbf{v}, \\
\rho \frac{D \mathbf{v}}{D t} & =\nabla \cdot \mathbf{T}-\rho \mathbf{g}, \\
\rho \frac{D \varepsilon}{D t} & =\mathbf{T}: \nabla \mathbf{v}-\nabla \cdot \mathbf{q} .
\end{aligned}
$$

Here $\mathbf{T}$ is the Cauchy stress tensor, $\rho$ is the density, $\mathbf{v}$ is the velocity, $\varepsilon$ is the specific internal energy density, $-\mathbf{g}$ is the gravitational acceleration, $\mathbf{q}$ is the heat flux, and $D / D t=\partial / \partial t+\mathbf{v} \cdot \nabla$.

To account for the influence of density gradients on energy in a manner compatible with the second law of thermodynamics, we apply a theory for Korteweg-type fluids described by J. E. Dunn and J. Serrin [9]. A recent review of related theories and their applications to diffuse-interface modeling has been given by Anderson, McFadden, and Wheeler [1]. Dunn and Serrin replace the energy balance equation with

$$
\rho \frac{D \varepsilon}{D t}=\mathbf{T}: \nabla \mathbf{v}-\nabla \cdot \mathbf{q}+\nabla \cdot \mathbf{u}
$$


where $\nabla \cdot \mathbf{u}$ represents a contribution made to the power by capillary effects due to the strong density gradients. They then postulate that $\varepsilon, \mathbf{T}, \mathbf{q}, \mathbf{u}$, as well as $\psi$, the specific Helmholtz free energy density, and $s$, the specific entropy density, are given by constitutive relations that depend only on the pointwise values of $\rho, T, \nabla \rho, \nabla \nabla \rho, \nabla T$, and $\nabla \mathbf{v}$, where $T$ is the temperature.

In order to guarantee that the equations of motion are compatible with the second law of thermodynamics as expressed by the Clausius-Duhem inequality, Dunn and Serrin deduce that the constitutive relations must be such that

$$
\psi=\bar{\psi}\left(\rho, T,|\nabla \rho|^{2}\right), \quad s=-\frac{\partial \bar{\psi}}{\partial T}, \quad \varepsilon=\bar{\psi}-T \frac{\partial \bar{\psi}}{\partial T}
$$

and

$$
\mathbf{u}=-\rho(\nabla \cdot \mathbf{v}) m \nabla \rho+\omega
$$

where $m=2 \rho(\partial \bar{\psi} / \partial M)(\rho, T, M)$ with $M=|\nabla \rho|^{2}$. Furthermore, in the case without viscosity, the stress must take the form

$$
\mathbf{T}=\left(-\rho^{2} \frac{\partial \bar{\psi}}{\partial \rho}+\rho \nabla \cdot(m \nabla \rho)\right) \mathbf{1}-m \nabla \rho \otimes \nabla \rho .
$$

In (2.6), the quantity $\omega$ measures the "static" part of the capillary work flux $\mathbf{u}$. For a class of materials including those we shall consider, $\nabla \cdot \omega=0$; so $\omega$ has no effect on the energy balance equation and can be ignored.

We choose the simplest possible form for the specific Helmholtz free energy density $\psi$ that is consistent with Dunn and Serrin's theory. Namely, we suppose that $m$ is constant, and that

$$
\psi=\hat{\psi}(\rho, T)+\frac{m}{2 \rho}|\nabla \rho|^{2} .
$$

This yields an expression for total free energy that appears in van der Waals' theory of capillarity [28], for example. From (2.5) it follows that

$$
\varepsilon=\hat{\varepsilon}(\rho, T)+\frac{m}{2 \rho}|\nabla \rho|^{2}, \quad s=-\frac{\partial \hat{\psi}}{\partial T}(\rho, T),
$$

where $\hat{\varepsilon}=\hat{\psi}-T(\partial \hat{\psi} / \partial T)$. We assume that the heat flux is given by $\mathbf{q}=-\kappa \nabla T$, with thermal conductivity coefficient $\kappa=\hat{\kappa}(\rho, T)$. We suppose that the stress is given by (2.7) with the addition of Newtonian viscosity terms, so that

$$
\mathbf{T}=\left(-p+\frac{m}{2}|\nabla \rho|^{2}+m \rho \Delta \rho\right) \mathbf{1}-m \nabla \rho \otimes \nabla \rho+\lambda(\nabla \cdot \mathbf{v}) \mathbf{1}+2 \mu \mathbf{D}
$$

where the pressure $p$ is given by

$$
p=\hat{p}(\rho, T)=\rho^{2} \frac{\partial \hat{\psi}}{\partial \rho}(\rho, T),
$$

$\mathbf{D}=\frac{1}{2}\left(\nabla \mathbf{v}+\nabla \mathbf{v}^{\mathrm{T}}\right)$ is the symmetric part of $\nabla \mathbf{v}$, and the viscosity coefficients have the form $\lambda=\hat{\lambda}(\rho, T), \mu=\hat{\mu}(\rho, T)$. For compatibility with the Clausius-Duhem inequality [9], one requires that $\lambda+\frac{2}{3} \mu \geq 0$. 
When Eqs. (2.6), (2.10), (2.8), and (2.9) are substituted into the balance laws (2.1), (2.2), (2.4), the result is the following system of equations for a viscous, heat-conducting fluid with capillary stresses:

$$
\begin{aligned}
\rho \frac{D \mathbf{v}}{D t}+\nabla p= & -\rho \mathbf{g}+m \rho \nabla \Delta \rho+\nabla(\lambda \nabla \cdot \mathbf{v})+\nabla \cdot(2 \mu \mathbf{D}), \\
\frac{D T}{D t}= & \left(1-\frac{c_{v}}{c_{p}}\right) \frac{K_{T}}{\alpha_{p}} \frac{D p}{D t}+\left(\rho c_{p}\right)^{-1} \nabla \cdot(\kappa \nabla T) \\
& +\left(\rho c_{p}\right)^{-1}\left(2 \mu \mathbf{D}: \mathbf{D}+\lambda(\nabla \cdot \mathbf{v})^{2}\right), \\
K_{T} \frac{D p}{D t}= & \alpha_{p} \frac{D T}{D t}-\nabla \cdot \mathbf{v} .
\end{aligned}
$$

Here $K_{T}=\rho^{-1}(\partial \rho / \partial p)_{T}$ is the isothermal compressibility, $\alpha_{p}=-\rho^{-1}(\partial \rho / \partial T)_{p}$ is the isobaric thermal expansion coefficient, and

$$
c_{v}=\hat{c}_{v}(\rho, T)=\frac{\partial \hat{\varepsilon}}{\partial T}, \quad c_{p}=\hat{c}_{p}(\rho, T)=c_{v}+\rho^{-1} \alpha_{p} T \frac{\partial \hat{p}}{\partial T}
$$

are the specific heat capacities at constant volume and at constant pressure, respectively. The system (2.12)-(2.14) differs from the standard system for a viscous, heat-conducting fluid by the addition of the single term $m \rho \nabla \Delta \rho$ in the momentum equation.

\section{Equations for slow flows with weak gravity.}

3.1. Scaling for the flow regime. In order to see how the system of equations (2.12)(2.14) can be appropriately simplified, we non-dimensionalize and scale the variables in a manner appropriate to a typical experiment, as follows. The critical density, critical pressure, and critical temperature are denoted $\rho_{c}, p_{c}$, and $T_{c}$, respectively. Let $x_{a}$ be a characteristic length, $t_{a}$ a characteristic time, and $v_{a}=x_{a} / t_{a}$. Let $c_{p_{a}}, c_{v_{a}}$ be characteristic specific heat capacities at constant pressure and at constant volume, respectively, and let $\Gamma=c_{p_{a}} / c_{v_{a}}$. Let $\kappa_{a}$ be a characteristic thermal conductivity, and let $\lambda_{a}, \mu_{a}$ be characteristic viscosity coefficients (for convenience we assume $\lambda_{a}=\mu_{a}$ ). Let $g_{a}=x_{a} / t_{a}^{2}$, and introduce the following non-dimensional variables and constants (non-dimensional quantities are indicated by an asterisk, $\left.{ }^{*}\right)$ :

$$
\begin{gathered}
\rho=\rho_{c} \rho^{*}, \quad p=p_{c}\left(1+p_{a} p^{*}\right), \quad T=T_{c}\left(1+T_{a} T^{*}\right), \\
\mathbf{x}=x_{a} \mathbf{x}^{*}, \quad t=t_{a} t^{*}, \quad \mathbf{v}=v_{a} \mathbf{v}^{*}, \quad \mathbf{g}=g_{a} \mathbf{g}^{*}, \\
c_{v}=c_{v_{a}} c_{v}^{*}, \quad c_{p}=c_{p_{a}} c_{p}^{*}, \quad \kappa=\kappa_{a} \kappa^{*}, \quad \lambda=\lambda_{a} \lambda^{*}, \quad \mu=\mu_{a} \mu^{*}, \\
K_{T}^{*}=\frac{1}{\rho^{*}}\left(\frac{\partial \rho^{*}}{\partial p^{*}}\right)_{T^{*}}, \quad \alpha_{p}^{*}=-\frac{1}{\rho^{*}}\left(\frac{\partial \rho^{*}}{\partial T^{*}}\right)_{p^{*}} .
\end{gathered}
$$

Here, $p_{a}$ and $T_{a}$ are non-dimensional scales characterizing the deviation of the pressure and temperature from critical. One tries to choose the scales so that the dimensionless variables $p^{*}, T^{*}, \mathbf{v}^{*}$ and their derivatives are of the order of 1 in the flow regime of interest . 
The non-dimensional equations corresponding to Eqs. (2.12)-(2.14) for a viscous fluid are

$$
\begin{aligned}
& \rho^{*} \frac{D \mathbf{v}^{*}}{D t^{*}}=-M^{-2} \nabla p^{*}+c \rho^{*} \nabla \Delta \rho^{*}-\rho^{*} \mathbf{g}^{*} \\
&+\operatorname{Re}^{-1}\left(\nabla\left(\lambda^{*} \nabla \cdot \mathbf{v}^{*}\right)+\nabla \cdot\left(2 \mu^{*} \mathbf{D}^{*}\right)\right), \\
& \frac{D T^{*}}{D t^{*}}=\left(1-\Gamma^{-1} \frac{c_{v}^{*}}{c_{p}^{*}}\right) \frac{K_{T}^{*}}{\alpha_{p}^{*}} \frac{D p^{*}}{D t^{*}}+\frac{D_{T}}{\rho^{*} c_{p}^{*}} \nabla \cdot\left(\kappa^{*} \nabla T^{*}\right) \\
& \quad+S\left(\frac{2 \mu^{*}}{\rho^{*} c_{p}^{*}} \mathbf{D}^{*}: \mathbf{D}^{*}+\frac{\lambda^{*}}{\rho^{*} c_{p}^{*}}\left(\nabla \cdot \mathbf{v}^{*}\right)^{2}\right), \\
& K_{T}^{*} \frac{D p^{*}}{D t^{*}}=\alpha_{p}^{*} \frac{D T^{*}}{D t^{*}}-\nabla \cdot \mathbf{v}^{*},
\end{aligned}
$$

where the gradient and the divergence are taken with respect to the non-dimensional spatial variable, and $D / D t^{*}=\partial / \partial t^{*}+\mathbf{v}^{*} \cdot \nabla$.

The dimensionless constants $M^{2}, D_{T}, c, \mathrm{Re}^{-1}$, and $S$ are defined by

$$
M^{2}=\frac{\rho_{c} v_{a}^{2}}{p_{c} p_{a}}, \quad D_{T}=\frac{\kappa_{a} t_{a}}{\rho_{c} c_{p_{a}} x_{a}^{2}}, \quad c=\frac{m \rho_{c} t_{a}^{2}}{x_{a}^{4}}, \quad \operatorname{Re}^{-1}=\frac{\mu_{a} t_{a}}{\rho_{c} x_{a}^{2}}, \quad S=\frac{\mu_{a}}{\rho_{c} c_{p_{a}} T_{c} T_{a} t_{a}} .
$$

The parameter $M$ is proportional to the Mach number $v_{a} / c_{s}$, where the sound speed is given by $c_{s}^{2}=(\partial p / \partial \rho)_{s}=c_{p} /\left(c_{v} \rho K_{T}\right)$. Re is the Reynolds number, $D_{T}$ is a nondimensional diffusivity, and $c$ is a non-dimensional coefficient of capillarity.

A typical flow regime in which we are interested is one considered in the paper by Boukari, Pego, and Gammon [5]. The fluid is xenon, with critical parameters

$$
\rho_{c}=1.11 \times 10^{3} \frac{\mathrm{kg}}{\mathrm{m}^{3}}, \quad p_{c}=5.84 \times 10^{6} \mathrm{~Pa}, \quad T_{c}=289.72 \mathrm{~K} .
$$

A typical experimental cell is about half a centimeter in radius; so we choose $x_{a}=10^{-3}$ $\mathrm{m}$. The time scale of interest ranges from a fraction of a second to hours. For now we take $t_{a}=1 \mathrm{~s}$ and postpone further discussion. The effect of earth's gravity tends to become important within about $30 \mathrm{mK}$ of $T_{c}^{*}$; so we take $T_{a}=10^{-4}$. In this temperature range and near the critical density, an appropriate model equation of state is the restricted cubic model, with coefficients for xenon (see [22] and the references therein). From this model, as in [5] we find it appropriate to take

$$
p_{a}=6 T_{a}, \quad c_{p_{a}} \approx 3.3 \times 10^{6} \frac{\mathrm{J}}{\mathrm{kg} \cdot K}, \quad \Gamma^{-1} \approx 1.8 \times 10^{-4} .
$$

We have not introduced a separate scale for deviations of the density from critical because these can be rather large, of the order of $10 \%$.

We estimate a characteristic value for thermal conductivity as in [5], using the approach described in $[31,29]$ to calculate the background term and divergent part near the critical point. To estimate the viscosity, we use the results of [31, Table III], also see [26]. As a result we find it appropriate to take

$$
\kappa_{a} \approx 2.5 \times 10^{-1} \frac{\mathrm{J}}{\mathrm{s} \cdot \mathrm{m} \cdot \mathrm{K}}, \quad \mu_{a} \approx 5 \times 10^{-5} \mathrm{~Pa} \cdot \mathrm{s} .
$$

Finally, we estimate the constant capillarity coefficient $m$ using the power law representation $m \sim \xi^{2} / \chi_{T}$ derived by Rowlinson and Widom [28], where the correlation 
length $\xi$ and susceptibility $\chi_{T}=\rho^{2} K_{T}$ at the critical density are given [30] by the power laws

$$
\xi \sim \xi_{0}\left|\Delta T^{*}\right|^{-\nu}, \quad \chi_{T} \sim \frac{\left(\rho_{c}\right)^{2} C}{p_{c}}\left|\Delta T^{*}\right|^{-\gamma} .
$$

Here $\Delta T^{*}=\left(T-T_{c}\right) / T_{c}=T_{a} T^{*} \approx 10^{-4}$, and we use from [30] the critical exponents $\gamma=1.19$ and $\nu=0.63$, and for xenon in the range $T>T_{c}$ approximately $\xi_{0}=1.9 \times 10^{-10}$, $C=.0813$. Using these values we obtain the estimate

$$
m \approx \frac{\left(5.84 \times 10^{6}\right)\left(1.9 \times 10^{-10}\right)^{2}}{\left(1.11 \times 10^{3}\right)^{2}(.0813)}\left(10^{-4}\right)^{-.07} \approx 4 \times 10^{-18} .
$$

From the estimates above, we obtain the following estimates for the dimensionless parameters:

$$
\begin{gathered}
M^{2} \approx 3 \times 10^{-7}, \quad D_{T} \approx 6 \times 10^{-5}, \quad c \approx 4.4 \times 10^{-3} \\
\operatorname{Re}^{-1} \approx 4.5 \times 10^{-2}, \quad S \approx 5 \times 10^{-13}
\end{gathered}
$$

and we find

$$
\left|\mathbf{g}^{*}\right|=9.81 \times 10^{3}, \quad \frac{c_{p}^{*}}{c_{v}^{*}} \sim 1, \quad\left(\frac{\partial p^{*}}{\partial T^{*}}\right)_{\rho^{*}} \sim 1 .
$$

The second speed $c_{s} \approx 80 \mathrm{~m} / \mathrm{s}$.

Regarding these parameters, several points are worthy of comment. First, note the effect of considering longer time scales. As $t_{a}$ increases, $S$ decreases and $M^{2}$ decreases quadratically, $D_{T}$ and $\mathrm{Re}^{-1}$ increase, and $c$ and $\mathrm{g}^{*}$ increase quadratically. Second, experiments performed in low earth orbit are reported to experience typical accelerations of $10^{-4}$ to $10^{-6}$ times earth's gravity [22]; this would make $\mathbf{g}^{*}$ of order 1 . (Another way to obtain $\mathbf{g}^{*}$ of order 1 is to consider a faster time scale like $t_{a}=.01 \mathrm{~s}$.) Also, we note that the value of $c$ becomes of order 1 when the characteristic length is replaced by a capillary length $x_{\text {cap }}$ for which $1=t_{a}^{2} m \rho_{c} / x_{\text {cap }}^{4}$. For our flow regime, we estimate $x_{\text {cap }} \approx x_{a}\left(4.4 \times 10^{-3}\right)^{1 / 4} \approx 260$ microns. In the recent ZENO experiment, observations were performed using light scattering through a fluid layer 100 microns thick [13].

We should also comment on the effect of proximity to the critical temperature. As $T_{a}$ approaches zero, we have seen that the capillary coefficient diverges very weakly, with exponent $\gamma-2 \nu \approx-0.07$. The viscosity and the sound speed also diverge at a very slow rate, changing only modestly over the experimental range of interest. The specific heat $c_{v}$ also diverges weakly, with exponent $-\alpha \approx-0.11$. The compressibility, thermal expansivity, and specific heat $c_{p}=c_{v}+\chi_{T} T \rho^{-3}(\partial \hat{p} / \partial T)^{2}$ diverge strongly, all with exponent $-\gamma \approx-1.19$. The thermal conductivity diverges less strongly, like $c_{p} / \xi \mu[30, \mathrm{p}$. $22]$, with approximately the exponent $-(\gamma-\nu) \approx-0.56$. So we see that as $T_{a}$ approaches zero, none of the non-dimensional constants above has a very strong dependence on $T_{a}$, though we can expect the non-dimensional diffusivity $D_{T}$ and $S$ to decrease.

3.2. Reduced equations for slow flows. To obtain simplified equations for compressible flows, we proceed formally in a manner motivated by the treatments of Rehm and Baum [27] and Majda and Sethian [21]. Since we are interested in longer time scales, we regard $M^{2}$ and $S$ as small and let $p_{0}, T_{0}, \mathbf{v}_{0}$ denote assumed asymptotic limits of $p^{*}, T^{*}, \mathbf{v}^{*}$, respectively, as $M^{2}$ and $S$ are taken to zero. In this process we regard $\mathbf{g}^{*}$ as fixed and of 
order one, corresponding to a low-gravity environment for our flow regime. Multiplying the momentum equation (3.1) by $M^{2}$ and taking $M^{2}$ to 0 , we require

$$
\nabla p_{0}\left(\mathbf{x}^{*}, t^{*}\right)=0
$$

and therefore $p_{0}=p_{0}\left(t^{*}\right)$ is constant in space. From (3.2)-(3.3), the asymptotic equations for temperature and pressure are

$$
\begin{aligned}
\frac{D T_{0}}{D t^{*}} & =\left(1-\Gamma^{-1} \frac{c_{v 0}}{c_{p 0}}\right) \frac{K_{T 0}}{\alpha_{p 0}} \frac{d p_{0}}{d t^{*}}+\frac{D_{T}}{\rho_{0} c_{p 0}} \nabla \cdot\left(\kappa_{0} \nabla T_{0}\right), \\
K_{T 0} \frac{d p_{0}}{d t^{*}} & =\alpha_{p 0} \frac{D T_{0}}{D t^{*}}-\nabla \cdot \mathbf{v}_{0} .
\end{aligned}
$$

Here the asymptotic density $\rho_{0}\left(\mathbf{x}^{*}, t^{*}\right)=\hat{\rho}^{*}\left(p_{0}\left(t^{*}\right), T_{0}\left(\mathbf{x}^{*}, t^{*}\right)\right)$ from the equation of state, and elsewhere the subscript 0 indicates a non-dimensional coefficient that is evaluated at $\left(\rho_{0}, T_{0}\right)$, e.g., $\kappa_{0}\left(\mathbf{x}^{*}, t^{*}\right)=\kappa^{*}\left(\rho_{0}, T_{0}\right)$. Equation (3.7) implies the mass continuity equation $D \rho_{0} / D t^{*}+\rho_{0} \nabla \cdot \mathbf{v}_{0}=0$.

Next we derive a reduced momentum equation. We suppose that the flow occurs in a bounded domain $\Omega$ with $\mathbf{v}=0$ on the boundary $\partial \Omega$. We use the fact that every square-integrable vector field $\mathbf{v}$ has a unique orthogonal decomposition of the form

$$
\mathbf{v}=\mathbf{w}+\nabla \phi, \quad \text { where } \nabla \cdot \mathbf{w}=0 \text { and }\left.\mathbf{w} \cdot \mathbf{n}\right|_{\partial \Omega}=0
$$

( $\mathbf{n}$ is the outward unit normal to $\partial \Omega$ ). We write $P \mathbf{v}=\mathbf{w}$; so $P$ denotes the orthogonal projection of square-integrable vector fields onto solenoidal vector fields. Observe that if $P \mathbf{f}=\mathbf{0}$, then $\mathbf{f}=\nabla h$ for some function $h$, and conversely. Applying $P$ to the momentum equation (3.1), then, eliminates the term of order $M^{-2}$. Taking $M^{2}$ to 0 produces

$$
0=P\left[-\rho_{0} \frac{D \mathbf{v}_{0}}{D t^{*}}-\rho_{0} \mathbf{g}^{*}+c \rho_{0} \nabla \Delta \rho_{0}+\frac{1}{\operatorname{Re}}\left(\nabla \cdot\left(\mu_{0}\left(\nabla \mathbf{v}_{0}+\nabla \mathbf{v}_{0}^{\mathrm{T}}\right)\right)+\nabla\left(\lambda_{0} \nabla \cdot \mathbf{v}_{0}\right)\right)\right]
$$

where $\mu_{0}=\mu^{*}\left(\rho_{0}, T_{0}\right), \lambda_{0}=\lambda^{*}\left(\rho_{0}, T_{0}\right)$.

From this we infer that there must be a scalar function $p_{1}=p_{1}\left(\mathbf{x}^{*}, t^{*}\right)$ such that the expression above in brackets equals $\nabla p_{1}$, that is,

$$
\rho_{0} \frac{D \mathbf{v}_{0}}{D t^{*}}+\nabla p_{1}=-\rho_{0} \mathbf{g}^{*}+c \rho_{0} \nabla \Delta \rho_{0}+\frac{1}{\operatorname{Re}}\left(\nabla \cdot\left(\mu_{0}\left(\nabla \mathbf{v}_{0}+\nabla \mathbf{v}_{0}^{\mathrm{T}}\right)\right)+\nabla\left(\lambda_{0} \nabla \cdot \mathbf{v}_{0}\right)\right) .
$$

Note that at this point we do not presume that the pressure $p^{*} \approx p_{0}+M^{2} p_{1}$ to order $M^{2}$. Klainerman and Majda [16] have found that at order $M^{2}$ there is an acoustic correction to pressure that depends on fast time and space scales.

Equations (3.6), (3.7), and (3.9) are the simplified equations for a viscous fluid. In order that our approximations be self-consistent, we must require that at the initial time, as $M^{2}$ and $S$ tend to zero we have

$$
p^{*}\left(\mathbf{x}^{*}, 0\right) \rightarrow p_{0}(0), \quad T^{*}\left(\mathbf{x}^{*}, 0\right) \rightarrow T_{0}\left(\mathbf{x}^{*}, 0\right), \quad \mathbf{v}^{*}\left(\mathbf{x}^{*}, 0\right) \rightarrow \mathbf{v}_{0}\left(\mathbf{x}^{*}, 0\right) .
$$

3.3. Reformulation. The full system (2.12)-(2.14) is appropriate for describing compressible fluid flow on acoustic time scales. In the flow regime for xenon that we have described, such time scales are short, since the sound speed is of the order of tens of meters per second. The simplified system (3.6), (3.7), (3.9) represents an "acoustic filtering" of the full system that describes flow on time scales that are long compared to acoustic. The pressure is maintained spatially constant through a process mediated by sound wave 
propagation. (An asymptotic description of this process for a near-critical van der Waals fluid was given by Zappoli and Carles [34] in one dimension with no viscosity.)

As is easy to check, both the full system and the simplified system are compatible with the Clausius-Duhem inequality

$$
\rho \frac{D s}{D t}+\nabla \cdot\left(\frac{\mathbf{q}}{T}\right) \geq 0
$$

We next reformulate the simplified system (3.6), (3.7), (3.9) into an equivalent form which better reveals its evolutionary character. This reformulation will be necessary for our future purpose of analyzing the initial-value problem. We will omit the subscripts and superscripts and return to dimensional quantities for notational convenience, writing

$$
\rho=\rho_{c} \rho_{0}, \quad \bar{p}=p_{c}\left(1+p_{a} p_{0}\right), \quad T=T_{c}\left(1+T_{a} T_{0}\right), \quad \mathbf{v}=v_{a} \mathbf{v}_{0} .
$$

We employ the decomposition $\mathbf{v}=\mathbf{w}+\nabla \phi$ described in (3.8). Substituting the temperature equation (3.6) into the pressure equation (3.7) and solving for $\nabla \cdot \mathbf{v}$, we obtain

$$
\nabla \cdot \mathbf{v}=\Delta \phi=-\frac{c_{v} K_{T}}{c_{p}} \frac{d \bar{p}}{d t}+\frac{\alpha_{p}}{\rho c_{p}} \nabla \cdot(\kappa \nabla T) .
$$

This elliptic equation has a solution with $\nabla \phi \cdot \mathbf{n}=\mathbf{v} \cdot \mathbf{n}=0$ on $\partial \Omega$ (and then $\nabla \phi$ is uniquely determined), if and only if a solvability condition holds, namely

$$
\int_{\Omega}\left(-\frac{c_{v} K_{T}}{c_{p}} \frac{d \bar{p}}{d t}+\frac{\alpha_{p}}{\rho c_{p}} \nabla \cdot(\kappa \nabla T)\right) d x=0 .
$$

Solving this equation for $d \bar{p} / d t$, we get

$$
\frac{d \bar{p}}{d t}=H(t):=\frac{\left.\int_{\Omega}\left(\alpha_{p} / \rho c_{p}\right) \nabla \cdot(\kappa \nabla T)\right) d x}{\int_{\Omega}\left(c_{v} K_{T} / c_{p}\right) d x} .
$$

This integro-differential equation is the pressure evolution equation. The remaining equations of the system can be written as

$$
\begin{aligned}
& \frac{D T}{D t}=\left(1-\frac{c_{v}}{c_{p}}\right) \frac{K_{T}}{\alpha_{p}} H(t)+\frac{1}{\rho c_{p}} \nabla \cdot(\kappa \nabla T), \\
& \rho \frac{D \mathbf{w}}{D t}=-\nabla \pi-\rho \mathbf{g}+c \rho \nabla \Delta \rho-\rho \frac{D(\nabla \phi)}{D t} \\
& \quad+\nabla \cdot\left(\mu\left(\nabla(\mathbf{w}+\nabla \phi)+\nabla(\mathbf{w}+\nabla \phi)^{\mathrm{T}}\right)\right)+\nabla(\lambda \Delta \phi), \\
& \Delta \phi=-\frac{c_{v} K_{T}}{c_{p}} H(t)+\frac{\alpha_{p}}{\rho c_{p}} \nabla \cdot(\kappa \nabla T), \\
& \nabla \cdot \mathbf{w}=0,
\end{aligned}
$$

along with the equation of state $\rho=\hat{\rho}(\bar{p}, T)$. (Here $\pi=p_{c} p_{a} M^{2} p_{1}$.)

From (3.15) evaluated at time $t=0$, we obtain the compatibility condition

$$
\left.\Delta \phi\right|_{t=0}=\left.\left[-\frac{c_{v} K_{T}}{c_{p}} H(t)+\frac{\alpha_{p}}{\rho c_{p}} \nabla \cdot(\kappa \nabla T)\right]\right|_{t=0} .
$$

This equation imposes a constraint on the gradient part $\nabla \phi(\mathbf{x}, 0)$ of the initial velocity field $\mathbf{v}(\mathbf{x}, 0)$, and leaves freedom for the choice of the initial solenoidal component $\mathbf{w}(\mathbf{x}, 0)$, as long as $\nabla \cdot \mathbf{w}(\mathbf{x}, 0)=0$. 
As we show in [8], to solve the initial-value problem for these equations, it is appropriate to specify initial data for the leading-order pressure, temperature, and divergence-free part of the velocity field:

$$
\bar{p}(0)=\bar{p}_{0}, \quad T(\mathbf{x}, 0)=T_{0}(\mathbf{x}), \quad \mathbf{w}(\mathbf{x}, 0)=\mathbf{w}_{0}(\mathbf{x})
$$

where $\nabla \cdot \mathbf{w}_{0}=0$. Initial data for the velocity will take the form

$$
\mathbf{v}(\mathbf{x}, 0)=\mathbf{v}_{0}(\mathbf{x})=\mathbf{w}_{0}(\mathbf{x})+\nabla \phi_{0}(\mathbf{x})
$$

where $\nabla \phi_{0}$ is determined by the compatibility condition (3.17).

3.4. The adiabatic time scale. We conclude this section by indicating how the simplified equations (3.12)-(3.16), in non-dimensional form, can be used to very roughly estimate the time scale $t_{1}$ for the adiabatic mechanism described in the introduction to produce a rapid bulk temperature response to boundary heating.

The non-dimensional form of $(3.12)$ is

$$
\frac{d p_{0}}{d t^{*}}=\frac{\left.\Gamma D_{T} \int_{\Omega}\left(\alpha_{p_{0}} /\left(\rho_{0} c_{p 0}\right)\right) \nabla \cdot\left(\kappa_{0} \nabla T_{0}\right)\right) d x}{\int_{\Omega}\left(c_{v 0} K_{T 0} / c_{p 0}\right) d x} .
$$

We consider a homogeneous fluid initially at equilibrium, whose boundary temperature is raised rapidly. As the boundary temperature is changed, a thin thermal boundary layer is created next to the wall. The width of this layer increases with time through heat diffusion, so is roughly given by $\sqrt{t^{*} D_{T}}$ using (3.6). In the boundary layer, we may roughly approximate the non-dimensional temperature $T_{0}$ by a function of the form $f\left(s / \sqrt{t^{*} D_{T}}\right)$, where $s$ is the distance to the boundary. Treating the coefficients in (3.20) as constant, we estimate $\Delta T_{0} \approx\left(t^{*} D_{T}\right)^{-1} f^{\prime \prime}\left(s / \sqrt{t^{*} D_{T}}\right)$ in the boundary layer. The integrand in the numerator is then of order $\left(t^{*} D_{T}\right)^{-1}$ in the boundary layer and zero elsewhere, and the integrand in the denominator is of order 1. Suppose the fluid domain is a cube with dimensional side length $L=x_{a} L^{*}$. Then the order of $d p_{0} / d t^{*}$ is given by

$$
\frac{d p_{0}}{d t^{*}} \sim \Gamma D_{T} \frac{6 L^{* 2} \sqrt{t^{*} D_{T}}\left(t^{*} D_{T}\right)^{-1}}{L^{* 3}}=\left(\frac{36 \Gamma^{2} D_{T}}{L^{* 2} t^{*}}\right)^{1 / 2} \text {. }
$$

The time integral of this expression produces an order-one change in $p_{0}$ (hence in $T_{0}$ ), when $t=t_{1}=t^{*} t_{a}=L^{2} \Gamma^{-2} / 144 D$, where $D=\kappa_{a} /\left(\rho_{c} c_{p_{a}}\right)$ is the characteristic thermal diffusivity. For the fluid parameters corresponding to the flow regime that we have described above, in a cell with side length $L=10^{-2} \mathrm{~m}$ we estimate $t_{1} \approx 3.3 \times 10^{-4} \mathrm{~s}$.

This time is longer but not much longer than the acoustic time $t_{c}$ for a sound wave to cross the cell, given by $t_{c}=L / c_{s} \approx 1.25 \times 10^{-4} \mathrm{~s}$. It is unrealistic to expect, however, that the boundary temperature can be raised so rapidly in experiment. So what this estimate indicates is that for processes in which the boundary temperature varies slowly compared to the acoustic time, the boundary-to-bulk coupling provided by the integro-differential equation for the mean pressure is efficient in effecting bulk temperature changes.

The above estimate for $t_{1}$ is consistent with the results of Onuki and Ferrell [24], except for the geometric factor of 144 appearing in the denominator. We expect that diffusion dominates the equilibration at approximately the time $t_{d}=L^{2} / 144 D \approx 10^{4} \mathrm{~s}$. Onuki, Hao, and Ferrell [25] characterize the intermediate regime between the long times 
$t / t_{1} \geq \Gamma^{2}$ and the short times $t / t_{1}=O(1)$ by the geometrical mean $t_{\text {int }} / t_{1} \equiv \Gamma$ so that $t_{\text {int }}=\Gamma t_{1}$. For our flow regime we estimate $t_{\text {int }} \approx 2 \mathrm{~s}$.

\section{Multi-dimensional flows with strong gravity.}

4.1. Motivation. In this section, we re-examine the equations of motion in the case of strong gravity. Motivating us is the problem of describing near-critical fluid flows and equilibration in earth's gravity. Recall that $\left|\mathbf{g}^{*}\right| \sim 10^{4}$ in the flow regime considered in Sec. 3.1 with earth's gravity. The simplified system (3.12)-(3.16) fails to capture some key features of equilibration in this situation.

In equilibrium, temperature is constant and density is stratified according to the basic equation of hydrostatic balance,

$$
\nabla p=-\rho \mathbf{g},
$$

and the equation of state. (We will neglect capillarity in most of this section.) Denoting equilibrium temperature by $T_{e}$ and density by $\rho_{e}(z)$, the equilibrium density gradient satisfies

$$
\frac{d \rho_{e}}{d z}(z)=-\chi_{T}\left(\rho_{e}(z), T_{e}\right) g
$$

As temperature approaches $T_{c}$, the critical temperature, $\chi_{T}\left(\rho_{c}, T_{e}\right)$, the susceptibility on the critical isochore, diverges as in (3.4). Thus the density gradient develops a singularity at the level of critical density, and the density profile becomes highly nonlinear. In Fig. 1 we plot density profiles for xenon in equilibrium at $1 \mathrm{G}$, using the restricted cubic equation of state as in [5].

For the simplified system (3.12)-(3.16), however, the density in equilibrium is constant, given by $\rho=\hat{\rho}\left(p_{e}, T_{e}\right)$. The hydrostatic balance $\nabla \pi=-\rho \mathbf{g}$ from (3.14) can be interpreted as supplying an $O\left(M^{2}\right)$ correction to the leading-order pressure. This correction is linear in $z$ and could be used to generate a density correction (by linearizing the equation of state, for example). But as it stands, the system (3.12)-(3.16) relies only on the leading-order density to determine the fluid flow. One can therefore expect this system to be inaccurate in modeling phenomena such as deep convection and gravity waves in near-equilibrium states.

In order to model related phenomena, researchers studying small-scale atmospheric circulations frequently approximate the continuity equation with the "anelastic" continuity equation

$$
\nabla \cdot(\bar{\rho} \mathbf{v})=0
$$

where $\bar{\rho}(z)$ is usually defined either as the density in an adiabatically stratified, horizontally-uniform reference state, or as the horizontally-averaged actual density. Batchelor [2] introduced an equation equivalent to (4.2). The name "anelastic" was given by Ogura and Phillips [23], who derived (4.2), together with approximate momentum and thermodynamic equations, through a systematic scale analysis. Important assumptions in their analysis are that: (i) all deviations $\delta \theta$ of the "potential temperature" from some constant mean value $\theta_{a}$ are small (this is equivalent to a similar statement for entropy variations), and (ii) the time scale of the disturbance is similar to the time scale for gravity wave oscillations. The terms neglected in their approximation are formally an order $\varepsilon=\delta \theta / \theta_{a}$ 


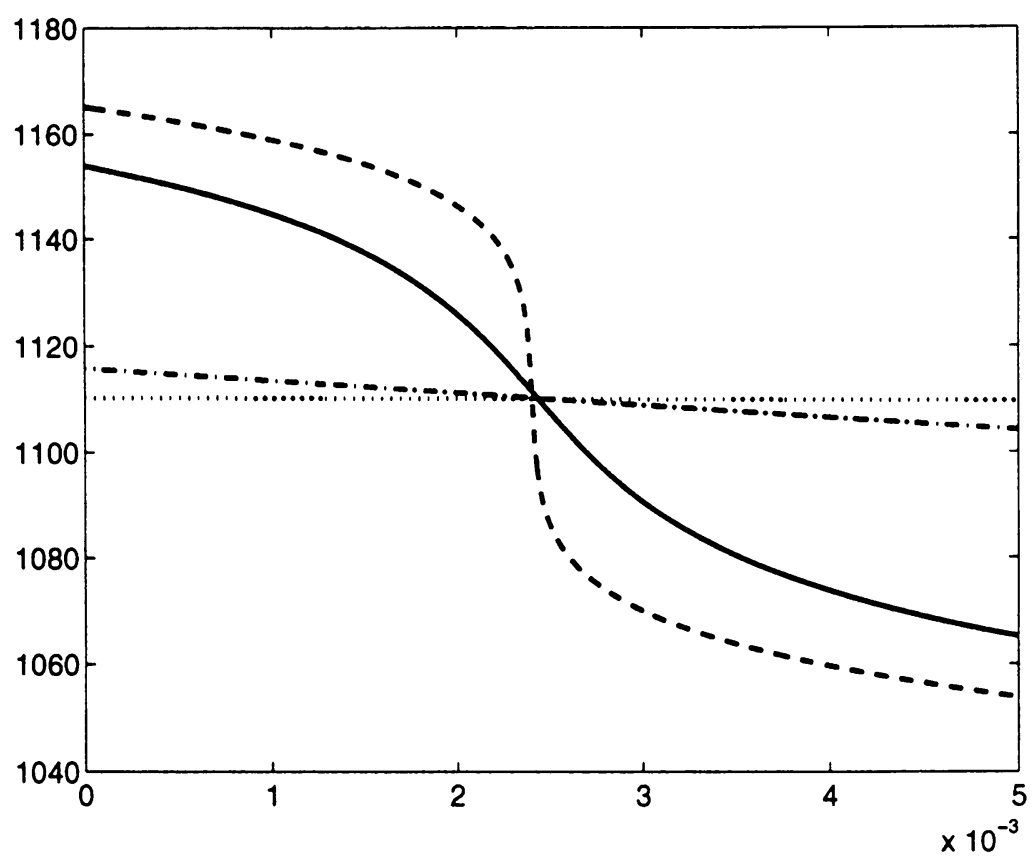

FIG. 1. Density versus height for near-critical xenon at 1G. $T-T_{c}$ in $\mathrm{mK}$ : 1 (dash), 10 (solid), 100 (dash-dot), 1000 (dot)

smaller than those that are retained. Their anelastic system does not support sound waves, does support gravity waves and conserves energy. Ogura and Phillips define $\bar{\rho}$ as the density in an adiabatically stratified, horizontally uniform reference state.

Some of the largest errors in the Ogura-Phillips anelastic approximation are reported to be generated by large deviations of the mean state potential temperature (or entropy) from a constant reference value. Several authors (Dutton and Fichtl [11], Wilhelmson and Ogura [32], Lipps and Hemler [19], Durran [10]) have presented alternative "sound-proof" equations in which thermodynamic variables associated with the adiabatic reference state are replaced with horizontally-uniform averaged or approximate actual values. These authors make different approximations in the momentum equations, but (except for Durran) they all obtain a continuity equation of the form (4.2) in which $\bar{\rho}$ is defined as a horizontally-averaged approximation to the actual density.

In the remainder of this section, we describe three possible models for multi-dimensional flows under strong gravity, making different assumptions about how to balance and approximate terms in the non-dimensionalized system (3.1)-(3.3):

(1) At first we scale so as to enforce hydrostatic balance at leading order. We find then that to be consistent, at leading order the thermodynamic variables must remain horizontally uniform. Also, the vertical velocity must remain horizontally uniform, unless we assume the entropy is spatially constant to leading order.

(2) If we indeed assume that entropy is spatially constant to leading order and also neglect heat conduction, we obtain a generalization of the anelastic approximation valid for a general equation of state. 
(3) Neither choice so far admits both thermodynamic equilibrium states and nonstratified multi-dimensional flows. We find, however, that if we return to the scaling adopted in Sec. 3.1, and modify the momentum equation so that the gravity force term incorporates a pressure correction self-consistently, then we get a system of the same formal accuracy that admits physically correct equilibrium states exactly. Moreover, the new system supports gravity waves but not acoustic waves.

4.2. Strongly stratified flow. Because equilibria are governed by the equation of hydrostatic balance, if gravity is strong it is natural to try to balance the pressure gradient term with the gravity term in the non-dimensional momentum equation (3.1). Thus we regard $M^{-2}$ and $\left|\mathbf{g}^{*}\right|$ to be of the same order. This same approach was taken in [5] for one-dimensional flows.

In this approximation, we rescale the gravitational acceleration, writing $\mathbf{g}^{*}=M^{-2} \overline{\mathbf{g}}$ where $\overline{\mathbf{g}}=O(1)$. It will prove instructive to replace $(p, T)$ by $(p, s)$ as the thermodynamic state variables, where $s$ is the specific entropy density. In terms of these variables, we write the equation of state as $\rho=\bar{\rho}(p, s)$. In non-dimensional form, we write $\rho^{*}=\tilde{\rho}^{*}\left(p^{*}, s^{*}\right)$, where entropy is non-dimensionalized via the relation $s=s_{a} s^{*}$. It is convenient to take $s_{a}=c_{p a} T_{a}$; this will be discussed further in the next subsection.

Starting from the non-dimensionalized system (3.1)-(3.3) with the replacement $\mathbf{g}^{*}=$ $M^{-2} \overline{\mathbf{g}}$, we proceed as in Sec. 3.2 and regard $M^{2}$ and $S$ as small, obtaining the temperature evolution equation (3.6) and continuity equation (3.7). In terms of the leading-order pressure $p_{0}$ and entropy $s_{0}$, these equations are equivalent to

$$
\begin{aligned}
\rho_{0}\left(1+T_{a} T_{0}\right) \frac{D s_{0}}{D t^{*}} & =D_{T} \nabla \cdot\left(\kappa \nabla T_{0}\right), \\
\frac{1}{\rho_{0} c_{s 0}^{2}} \frac{D p_{0}}{D t^{*}} & =\frac{\alpha_{p 0}}{\rho_{0} c_{p 0}}\left(\rho_{0}\left(1+T_{a} T_{0}\right) \frac{D s_{0}}{D t^{*}}\right)-\nabla \cdot \mathbf{v}_{0} .
\end{aligned}
$$

The leading-order terms in the momentum equation now just yield the equation of hydrostatic balance,

$$
\nabla p_{0}=-\rho_{0} \overline{\mathbf{g}}
$$

This equation imposes tight restrictions on the spatial dependence of the leading-order thermodynamic state variables. In particular, it is necessary that $p_{0}$ is a function only of height $z^{*}$ and time $t^{*}$ and is independent of the horizontal variables $\left(x^{*}, y^{*}\right)$. Then from (4.5) it follows that $\rho_{0}=\rho_{0}\left(z^{*}, t^{*}\right)$ is also horizontally uniform. The equation of state now implies that $s_{0}=s_{0}\left(z^{*}, t^{*}\right)$ as well. The entropy and pressure equations (4.3)-(4.4) now read

$$
\begin{aligned}
\rho_{0}\left(1+T_{a} T_{0}\right)\left(\frac{\partial s_{0}}{\partial t^{*}}+w_{0} \frac{\partial s_{0}}{\partial z^{*}}\right) & =D_{T} \frac{\partial}{\partial z^{*}}\left(\kappa_{0} \frac{\partial T_{0}}{\partial z^{*}}\right) \\
\frac{1}{\rho_{0} c_{s 0}^{2}}\left(\frac{\partial p_{0}}{\partial t^{*}}+w_{0} \frac{\partial p_{0}}{\partial z^{*}}\right) & =D_{T} \frac{\alpha_{p 0}}{\rho_{0} c_{p 0}} \frac{\partial}{\partial z^{*}}\left(\kappa_{0} \frac{\partial T_{0}}{\partial z^{*}}\right)-\nabla \cdot \mathbf{v}_{0}
\end{aligned}
$$

Here $w_{0}$ is the vertical component of the velocity $\mathbf{v}_{0}$.

If we assume $\partial s_{0} / \partial z^{*} \neq 0$, then to satisfy (4.6) consistently, the vertical velocity must be horizontally uniform: $w_{0}=w_{0}\left(z^{*}, t^{*}\right)$. We can eliminate the horizontal components of velocity from (4.7) by integrating over $\left(x^{*}, y^{*}\right)$; if the cell walls are vertical or periodic 
we can express the result in terms of density as the usual one-dimensional continuity equation,

$$
\frac{\partial \rho_{0}}{\partial t^{*}}+\frac{\partial\left(\rho_{0} w_{0}\right)}{\partial z^{*}}=0
$$

The system consisting of the three equations (4.5), (4.6), (4.8) is equivalent to the onedimensional system (14)-(16) in [5] for temperature and pressure, which was expressed using a Lagrangian variable $z^{\prime}=\int_{0}^{z^{*}} \rho_{0}\left(h, t^{*}\right) d h$ in place of the height $z^{*} \in[0, L]$. With $t^{\prime}=t^{*}$, in the present notation this system takes the form

$$
\begin{aligned}
p_{0}\left(z^{\prime}, t^{\prime}\right) & =p_{a}\left(t^{\prime}\right)-\bar{g} z^{\prime}, \\
\frac{\partial T_{0}}{\partial t^{\prime}} & =\left(1-\frac{c_{v 0}}{\Gamma c_{p 0}}\right) \frac{K_{T 0}}{\alpha_{p 0}} \frac{d p_{a}}{d t^{\prime}}+\frac{D_{T}}{c_{p 0}} \frac{\partial}{\partial z^{\prime}}\left(\rho_{0} \kappa_{0} \frac{\partial T_{0}}{\partial z^{\prime}}\right), \\
\frac{d p_{a}}{d t^{\prime}} & =\frac{\int_{0}^{m} \rho_{0}^{-1} \alpha_{p 0}\left(\partial T_{0} / \partial t^{\prime}\right) d z^{\prime}}{\int_{0}^{m} \rho_{0}^{-1} K_{T 0} d z^{\prime}} .
\end{aligned}
$$

(Here $m=\int_{0}^{L} \rho_{0}\left(h, t^{*}\right) d h$ is the total linear mass.) This system determines the evolution of the temperature, pressure, and vertical velocity. Vertical fluid motions in this model are due solely to density changes that occur in a horizontally stratified manner-the equations do not support acoustic or gravity waves.

The horizontal components of the momentum equation at order 1 govern the horizontal fluid flows within each material layer corresponding to $z^{\prime}=$ const. Presuming that $p_{1}$ yields an $O\left(M^{2}\right)$ correction to the leading-order pressure, we may write the horizontal components of the momentum equation as follows: Let $\mathbf{u}$ denote the horizontal components of velocity $\mathbf{v}_{0}$ and the subscript $h$ denote differentiation with respect to the horizontal variables $\mathbf{x}_{h}=\left(x^{*}, y^{*}\right)$. Then the horizontal momentum equation for $\mathbf{u}\left(\mathbf{x}_{h}, z^{\prime}, t^{\prime}\right)$ is

$$
\rho_{0}\left(\frac{\partial \mathbf{u}}{\partial t^{\prime}}+\left(\mathbf{u} \cdot \nabla_{h}\right) \mathbf{u}\right)+\nabla_{h} p_{1}=\frac{1}{\operatorname{Re}}\left(\mu_{0} \Delta_{h} \mathbf{u}+\rho_{0} \frac{\partial}{\partial z^{\prime}}\left(\rho_{0} \mu_{0} \frac{\partial \mathbf{u}}{\partial z^{\prime}}\right)\right) .
$$

Horizontal motions are incompressible, because $\nabla_{h} \cdot \mathbf{u}=0$ from (4.7) and (4.8). When viscosity can be neglected, then the equations corresponding to different fluid layers decouple, and fluid layers can exhibit arbitrary independent two-dimensional incompressible flows.

The vertical component of the momentum equation at order 1 can be used in determining higher-order corrections of order $M^{2}$ for horizontally-averaged density, pressure, and temperature. For the sake of brevity we omit further discussion.

4.3. The anelastic approximation. In order to admit convective flows with nontrivial vertical circulation and/or gravity waves, we can suppose that the entropy is spatially constant at leading order. As we have indicated, this is the same as the assumption made by Ogura and Phillips that potential temperature variations are small compared to a reference value.

At leading order, then, the fluid density, pressure, and temperature are in hydrostatic balance and adiabatically stratified, with a (negative) adiabatic temperature gradient. We must neglect heat conduction to maintain constant entropy; this restricts the time scale, and any flows generated will be adiabatic. 
To describe flows, we use the notation of Sec. 3.1 for non-dimensional and leadingorder quantities, and non-dimensionalize entropy according to $s=s_{a} s^{*}$ where $s_{a}=c_{p a} T_{a}$ (as discussed below). We postulate that the non-dimensional pressure $p^{*}$ and entropy $s^{*}$ are given to order $O\left(M^{2}\right)$ by

$$
p^{*} \sim p_{0}\left(z^{*}\right)+M^{2} p_{1}\left(\mathbf{x}^{*}, t^{*}\right), \quad s^{*} \sim s_{0}+M^{2} s_{1}\left(\mathbf{x}^{*}, t^{*}\right),
$$

where $s_{0}$ is a constant. (This neglects any fast-time acoustic corrections that may be present as discussed in [16].) Then we expect the density $\rho^{*} \sim \rho_{0}\left(z^{*}\right)+M^{2} \rho_{1}\left(\mathbf{x}^{*}, t^{*}\right)$, where from the equation of state,

$$
\rho_{1}=\left(\frac{\partial \rho^{*}}{\partial p^{*}}\right)_{s^{*}} p_{1}+\left(\frac{\partial \rho^{*}}{\partial s^{*}}\right)_{p^{*}} s_{1}=\frac{1}{c_{s 0}^{2}} p_{1}+\left(1+T_{a} T_{0}\right) \frac{\rho_{0} \alpha_{p 0}}{c_{p 0}} s_{1} .
$$

Here the non-dimensional coefficients are evaluated at $\left(p_{0}, s_{0}\right)$.

The momentum equation at order 1 is

$$
\rho_{0} \frac{D \mathbf{v}_{0}}{D t^{*}}+\nabla p_{1}=-\rho_{1} \overline{\mathbf{g}}+\frac{1}{\operatorname{Re}}\left(\nabla \cdot\left(\mu_{0}\left(\nabla \mathbf{v}_{0}+\nabla \mathbf{v}_{0}^{\mathrm{T}}\right)\right)+\nabla\left(\lambda_{0} \nabla \cdot \mathbf{v}_{0}\right)\right) .
$$

Since heat conduction is neglected, the entropy $s_{1}$ is convected with the flow, satisfying

$$
\frac{D s_{1}}{D t^{*}}=0 \text {. }
$$

Because $\partial \rho_{0} / \partial t^{*}=0$, the leading-order continuity equation yields

$$
\nabla \cdot\left(\rho_{0} \mathbf{v}_{0}\right)=0 .
$$

This constraint on the velocity should be used in solving (4.14) to determine $p_{1}$. Note, however, that $p_{1}$ will not be completely determined by the constraint-Given any solution of (4.14), $p_{1}$ can be replaced by adding an arbitrary time-dependent multiple of a solution to the linearized hydrostatic balance equation

$$
\frac{\partial \tilde{p}}{\partial z^{*}}=-\frac{\bar{g}}{c_{s 0}^{2}} \tilde{p}
$$

The equations (4.14)-(4.16) correspond to the anelastic equations of Ogura and Phillips, generalized for an arbitrary equation of state.

As a model for slow flows of fluids near the critical point, these equations have some drawbacks: first, heat conduction is entirely neglected; so the fast adiabatic mechanism for rapid thermal response is not accounted for within this model. Also, the effect of fluid flow on thermal relaxation cannot be evaluated. Moreover, describing states in thermodynamic equilibrium is problematic. At rest, the equations permit an arbitrary horizontally uniform entropy correction $s_{1}$. It may or may not be consistent with the derivation of the equations to take this to correspond to the equilibrium entropy profile (meaning the hydrostatic profile at constant temperature).

Regarding this point, we can ask, what is the size of the non-dimensional equilibrium entropy gradient in the regime of interest? To estimate this, we need to identify a typical entropy change in a process of interest. Consider a fluid at equilibrium at one temperature, subject to a temperature change at the boundary of order $T_{c} T_{a}$. During the early development of the thermal boundary layer, we may suppose roughly that the entropy change in the boundary layer occurs at constant pressure; so it is of order 
$s_{a}=c_{p a} T_{a}$ since $(\partial s / \partial T)_{p}=c_{p} / T$ and $T_{a}$ is small. Then it seems reasonable to nondimensionalize the entropy by letting $s=s_{a} s^{*}$.

Now, the equilibrium entropy gradient satisfies

$$
\frac{d s}{d z}=\left(\frac{\partial s}{\partial p}\right)_{T} \frac{d p}{d z}=\frac{c_{p}-c_{v}}{T}\left(\frac{\partial T}{\partial p}\right)_{\rho}(-\rho g) .
$$

Non-dimensionalizing this expression, we find that up to a quantity of order one,

$$
\frac{d s^{*}}{d z^{*}} \sim-M^{2}\left|\mathbf{g}^{*}\right|=-\frac{x_{a} g \rho_{c}}{p_{c} p_{a}}
$$

Under the specific conditions considered in Sec. 3.1, $d s^{*} / d z^{*} \sim-.003$. This is moderately small but not quite of order $M^{2}$; it would make $d s_{1} / d z^{*}$ of the order of $-10^{4}$. Also, since $p_{a} \approx 6 T_{a}$, the last member of (4.17) shows that $d s^{*} / d z^{*}$ diverges as temperature approaches critical, like $T_{a}^{-1}$ as $T_{a} \rightarrow 0$.

It seems that in our flow regime, the entropy gradient may not be small enough for the anelastic approximation to be reasonable for states near true equilibrium (at least at the level of critical density). But states near constant entropy do possess experimental interest. Physically, the condition that an inviscid stratified fluid is stable against convection is is $d s / d z<0$ [18]. In order to obtain "pseudo-equilibrium" states with near-uniform density profiles, ground-based experiments have been suggested on nearcritical fluids in which a steady-state heat flux is maintained so as to achieve a marginally stable entropy profile [22]; also see [4]. Note that the adiabatic density gradient satisfies $\rho^{-1} d \rho / d z=-g / c_{s}^{2}$, which is about $1 / 500 \mathrm{~m}^{-1}$ in our flow regime; so the density is close to constant in centimeter-sized cells. Provided that heat conduction can be neglected on the time scale of interest, the anelastic approximation could be useful, to describe flows near such "pseudo-equilibrium" states.

4.4. Slow flows including equilibrium. In trying to enforce hydrostatic balance at leading order, we have found that either flows remain strictly stratified, or equilibrium states are not admissible since entropy must be constant at leading order in $M^{2}$. In this section we return to the scaling as it was done in Sec. 3.2, in which the leading-order pressure turns out to be spatially constant. Working in the spirit of the Boussinesq approximation, we can decide to selectively retain some terms of higher order in $M^{2}$ where it would be most useful, without affecting the formal accuracy of the system.

We propose no alteration in the Eqs. (3.6)-(3.7) for leading-order temperature and mean pressure. (But now $p_{0}$ and $T_{0}$ will depend on $M^{2}$, through the coupling to the momentum equation.) To order $M^{2}$, supposing that the pressure is given by $p^{*} \sim$ $p_{0}+M^{2} p_{1}$, the approximation to the density $\rho \approx \rho_{0}=\hat{\rho}^{*}\left(p_{0}, T_{0}\right)$ can be improved to

$$
\rho \approx \check{\rho} \equiv \hat{\rho}^{*}\left(p_{0}+M^{2} p_{1}, T_{0}\right)
$$

We propose to make this improvement only in Eq. (3.9) for the velocity. The new system of equations governing leading-order temperature, pressure, and velocity (neglecting 
capillarity) is

$$
\begin{aligned}
\frac{D T_{0}}{D t^{*}} & =\left(1-\Gamma^{-1} \frac{c_{v 0}}{c_{p 0}}\right) \frac{K_{T 0}}{\alpha_{p 0}} \frac{d p_{0}}{d t^{*}}+\frac{D_{T}}{\rho_{0} c_{p 0}} \nabla \cdot\left(\kappa_{0} \nabla T_{0}\right), \\
K_{T 0} \frac{d p_{0}}{d t^{*}} & =\alpha_{p 0} \frac{D T_{0}}{D t^{*}}-\nabla \cdot \mathbf{v}_{0}, \\
\check{\rho} \frac{D \mathbf{v}_{0}}{D t^{*}}+\nabla p_{1} & =-\check{\rho} \mathbf{g}^{*}+\frac{1}{\operatorname{Re}}\left(\nabla \cdot\left(\mu_{0}\left(\nabla \mathbf{v}_{0}+\nabla \mathbf{v}_{0}^{\mathrm{T}}\right)\right)+\nabla\left(\lambda_{0} \nabla \cdot \mathbf{v}_{0}\right)\right) .
\end{aligned}
$$

The temperature evolution and continuity equations (4.18)-(4.19) may be replaced by the equivalent pair (4.3)-(4.4), in which the material derivative $D p_{0} / D t^{*}$ can be replaced by the ordinary derivative $d p_{0} / d t^{*}$.

As with the anelastic equations, the pressure correction $p_{1}$ should be determined in solving (4.20) to satisfy the implied constraint on the divergence of velocity from (4.19). The pressure correction will not be unique, but any two solutions $p_{1}$ and $\tilde{p}_{1}$ that correspond to the same $\left(p_{0}, T_{0}, \mathbf{v}_{0}\right)$ will be related by

$$
\nabla p_{1}+\hat{\rho}^{*}\left(p_{0}+M^{2} p_{1}, T_{0}\right) \mathbf{g}^{*}=\nabla \tilde{p}_{1}+\hat{\rho}^{*}\left(p_{0}+M^{2} \tilde{p}_{1}, T_{0}\right) \mathbf{g}^{*} .
$$

Therefore the difference $p_{1}-\tilde{p}_{1}$ is a function of $z^{*}$ and $t^{*}$ that is determined solely in terms of a function of $t^{*}$ by solving an ordinary differential equation.

The system consisting of Eqs. (4.18)-(4.20) can be reformulated to better reveal its evolutionary character exactly as in Sec. 3.3. In dimensional form with pressure $\vec{p}(t)+$ $\pi(x, t)$, where $\bar{p}=p_{c}\left(1+p_{a} p_{0}\right)$ and $\pi=p_{c} p_{a} M^{2} p_{1}$, one obtains exactly the system (3.12)-(3.16) except that (3.14) is replaced by

$$
\begin{aligned}
\check{\rho} \frac{D \mathbf{w}}{D t}= & -\nabla \pi-\check{\rho} \mathbf{g}-\check{\rho} \frac{D(\nabla \phi)}{D t} \\
& +\nabla \cdot\left(\mu\left(\nabla(\mathbf{w}+\nabla \phi)+\nabla(\mathbf{w}+\nabla \phi)^{\mathrm{T}}\right)\right)+\nabla(\lambda \Delta \phi),
\end{aligned}
$$

where the (now dimensional) density $\check{\rho}$ is determined from pressure and temperature by the equation of state: $\check{\rho}=\hat{\rho}(\bar{p}+\pi, T)$. We anticipate that, like system (3.12)-(3.16), solutions of (4.18)-(4.20) are determined by initial values for the temperature field, mean pressure, and divergence-free part of the velocity field.

The system (4.18)-(4.20) admits as rest states true equilibrium states with constant temperature and hydrostatic balance between the total pressure $p_{0}+M^{2} p_{1}$ and the improved density $\check{\rho}=\hat{\rho}^{*}\left(p_{0}+M^{2} p_{1}, T_{0}\right)$. It filters acoustic waves but admits gravity waves, as we shall show in the subsection to follow. Moreover, heat conduction need not be neglected; so the adiabatic effect can be modeled. It should be interesting to study which flows are generated when large density changes in the thermal boundary layer are present together with very stable equilibrium entropy profiles away from the boundary.

We remark that it is evidently not necessary to replace $\rho_{0}$ by $\check{\rho}$ in the acceleration term of (3.9) to gain true equilibria as rest states. In some circumstances it may be more convenient not to make this replacement. But it turns out that Eq. (4.20) is slightly more convenient when we study the linearized equations for gravity waves; see the next section.

The system (4.18)-(4.20) certainly has shortcomings. First, in the flow regime described in Sec. 3.1, $\left|\mathbf{g}^{*}\right|=g t_{a}^{2} / x_{a}$ is still rather large. This problem diminishes if a larger 
space scale or smaller time scale is relevant. The system can be expected to lose formal validity if solutions become large or singular, as may well happen in a nonadiabatic convection process. Another point is that, while the leading-order total energy is conserved in time for the weak-gravity equations (3.12)-(3.16), this is not strictly true for the system (4.18)-(4.20). The time derivative of the leading-order total energy is formally of order $M^{2}$ instead.

4.5. Gravity waves in the linear approximation. We wish to verify that in the linear approximation near a stably stratified rest state for which entropy decreases with height, the system (4.18)-(4.20) admits gravity waves but not acoustic waves when heat conduction and viscosity are neglected. We shall also show that, in the special case of a perfect gas with an exponential density profile and constant sound speed, in the limit of large wave number the gravity-wave frequency approaches the Brunt-Väisälä frequency $N$ corresponding to a compressible fluid. This frequency satisfies

$$
N^{2}=-\frac{g}{\rho_{e}} \frac{d \rho_{e}}{d z}-\frac{g^{2}}{c_{s}^{2}} .
$$

The second term does not appear in the usual treatment of gravity waves for a stratified incompressible fluid, in which the density is advected with the flow, cf. [33].

When heat conduction and viscosity are neglected in the system (4.18)-(4.20), the leading-order pressure is constant in time as well as space. Consequently, the temperature (and entropy) are advected with the flow, and the velocity field has zero divergence. In dimensional form with $p=\bar{p}+\pi$ and $\check{\rho}=\tilde{\rho}(p, s)$, the governing equations take the form

$$
\begin{aligned}
\frac{D s}{D t} & =0, \\
\nabla \cdot \mathbf{v} & =0, \\
\check{\rho} \frac{D \mathbf{v}}{D t}+\nabla p & =-\check{\rho} \mathbf{g} .
\end{aligned}
$$

Near a rest state where $(p, s, \mathbf{v})=\left(p_{e}(z), s_{e}(z), \mathbf{0}\right)$, we write

$$
p=p_{e}+\tilde{p}, \quad s=s_{e}+\tilde{s}, \quad \mathbf{v}=(\tilde{u}, \tilde{v}, \tilde{w})
$$

and $\rho_{e}(z)=\tilde{\rho}\left(p_{e}, s_{e}\right)$. Then we linearize, obtaining

$$
\begin{aligned}
\frac{\partial \tilde{s}}{\partial t}+\tilde{w} s_{e}^{\prime}(z) & =0 \\
\nabla \cdot \mathbf{v} & =0 \\
\rho_{e} \frac{\partial \mathbf{v}}{\partial t}+\nabla \tilde{p} & =-\left(\left(\frac{\partial \rho}{\partial p}\right)_{s} \tilde{p}+\left(\frac{\partial \rho}{\partial s}\right)_{p} \tilde{s}\right) \mathbf{g}
\end{aligned}
$$

where the coefficients are evaluated at $\left(p_{e}, s_{e}\right)$. Let us suppose that periodic boundary conditions are specified in the horizontal variables $(x, y)$. We look for normal modes with

$$
(\tilde{p}, \tilde{s}, \tilde{u}, \tilde{v}, \tilde{w})=e^{i\left(k_{1} x+k_{2} y-\omega t\right)}(\bar{p}, \bar{s}, \bar{u}, \bar{v}, \bar{w})(z)
$$


and eliminate $\bar{p}, \bar{s}$ and the horizontal components of velocity from the system. Note that the sound speed satisfies $c_{s}^{-2}=(\partial \rho / \partial p)_{s}$, and define

$$
\alpha(z)=-\frac{1}{\rho_{e}} \frac{d \rho_{e}}{d z}, \quad \beta(z)=-\frac{1}{\rho_{e}}\left(\frac{\partial \rho}{\partial s}\right)_{p} \frac{d s_{e}}{d z}=\alpha(z)-\frac{g}{c_{s}^{2}} .
$$

(The last identity holds due to hydrostatic balance.) Then for the vertical velocity component $\bar{w}(z)$ we obtain the equation

$$
-\frac{d^{2} \bar{w}}{d z^{2}}+\beta \frac{d \bar{w}}{d z}+\left(k^{2}-\frac{k^{2} \beta g}{\omega^{2}}\right) \bar{w}=0
$$

where $k^{2}=k_{1}^{2}+k_{2}^{2}$. The vertical velocity must vanish at the top and bottom boundaries. So, given a horizontal wave number $k$, possible oscillation frequencies $\omega$ are determined by solving the eigenvalue problem in (4.28) with Dirichlet boundary conditions.

We note that in the special case of perfect gas at constant temperature, the density profile is exponential and sound speed is constant, and so $\alpha(z)$ and $\beta(z)$ are constant. Then (4.28) has explicit solutions of the form $\bar{w}(z)=e^{\beta z / 2} \sin (n z)$, whence the gravitywave dispersion relation is given by

$$
\omega^{2}=\frac{k^{2} \beta g}{k^{2}+n^{2}+\frac{1}{4} \beta^{2}} .
$$

In the limit $k^{2} \rightarrow \infty$, this expression approaches $\beta g=N^{2}$, where $N$ from (4.23) is the Brunt-Väisälä frequency for a compressible fluid. For comparison, for a fully compressible fluid in which one starts with $D \check{\rho} / D t+\check{\rho} \nabla \cdot \mathbf{v}=0$ in place of $(4.25)$, the dispersion relation in this special case is

$$
\omega^{2}=\frac{c_{s}^{2}}{2}\left(k^{2}+n^{2}+\frac{\alpha^{2}}{4} \pm \sqrt{\left(k^{2}+n^{2}+\frac{\alpha^{2}}{4}\right)^{2}-4 k^{2} \frac{N^{2}}{c_{s}^{2}}}\right) .
$$

For large $k^{2}$, the plus sign yields $\omega^{2} \approx c_{s}^{2} k^{2}$, corresponding to acoustic waves, and the minus sign yields $\omega^{2} \approx N^{2}$, corresponding to gravity waves. The magnitude of any oscillation frequency $\omega$ that satisfies (4.29) is less than $N$, showing that the system (4.24)-(4.26) supports gravity waves but not acoustic waves.

In general, when the coefficients in (4.28) vary with $z$, we can obtain an upper bound on oscillation frequencies as follows. Let $q(z)=\exp \left(-\int^{z} \beta(\zeta) d \zeta\right)$, multiply equation (4.28) by $q \bar{w}$ and integrate over $z \in[0, L]$, from bottom to top. One obtains

$$
\int_{0}^{L} q(z) \bar{w}^{\prime}(z)^{2} d z+\frac{k^{2}}{\omega^{2}} \int_{0}^{L}\left(\omega^{2}-\beta g\right) q(z) \bar{w}(z)^{2} d z=0 .
$$

Since the second integrand cannot be everywhere nonnegative, it follows that

$$
\omega^{2}<\max _{z} \beta(z) g
$$

The dispersion relation in (4.29) is very similar to that obtained in the usual case of an incompressible fluid when one assumes the density is advected with the flow. Starting 
from the governing equations

$$
\begin{aligned}
\frac{D \rho}{D t} & =0, \\
\nabla \cdot \mathbf{v} & =0, \\
\rho \frac{D \mathbf{v}}{D t}+\nabla p & =-\rho \mathbf{g},
\end{aligned}
$$

one finds in similar fashion that the equation corresponding to (4.28) is

$$
-\frac{d^{2} \bar{w}}{d z^{2}}+\alpha \frac{d \bar{w}}{d z}+\left(k^{2}-\frac{k^{2} \alpha g}{\omega^{2}}\right) \bar{w}=0,
$$

and for an exponential density profile the dispersion relation is

$$
\omega^{2}=\frac{k^{2} \alpha g}{k^{2}+n^{2}+\frac{1}{4} \alpha^{2}} .
$$

In the limit $k^{2} \rightarrow \infty$ this approaches $\alpha g=N_{0}^{2}$, where $N_{0}$ is the usual Brunt-Väisälä frequency for an incompressible fluid. In many circumstances the difference between $N_{0}$ and $N$ may be negligible, but it is interesting that the dispersion relation arising from the system (4.24)-(4.26) more faithfully approximates the compressible case in this respect.

4.6. Final remarks. We close with a few remarks intended to clarify the differences between the new system (4.18)-(4.20) and the anelastic system (4.13)-(4.16). For purposes of comparison, we neglect heat conduction. In this case, the new system (4.3), (4.4), (4.20) becomes

$$
\begin{aligned}
\frac{D s_{0}}{D t^{*}} & =0, \\
\nabla \cdot \mathbf{v}_{0} & =0, \\
\check{\rho} \frac{D \mathbf{v}_{0}}{D t^{*}}+\nabla p_{1} & =-\check{\rho} \mathbf{g}^{*}+\frac{1}{\operatorname{Re}}\left(\nabla \cdot\left(\mu_{0}\left(\nabla \mathbf{v}_{0}+\nabla \mathbf{v}_{0}^{\mathrm{T}}\right)\right)+\nabla\left(\lambda_{0} \nabla \cdot \mathbf{v}_{0}\right)\right),
\end{aligned}
$$

where $\check{\rho}=\tilde{\rho}^{*}\left(p_{0}+M^{2} p_{1}, s_{0}\right)$.

For each system, the source of the constraint on velocity ((4.37) or (4.16) respectively) is the continuity equation

$$
\frac{D \rho_{0}}{D t^{*}}+\rho_{0} \nabla \cdot \mathbf{v}_{0}=0
$$

where $\rho_{0}=\tilde{\rho}^{*}\left(p_{0}, s_{0}\right)$. For the anelastic system, the leading-order entropy $s_{0}$ is constant and the pressure $p_{0}$ is a function of $z$ determined by the hydrostatic balance equation (4.5); so $D \rho_{0} / D t^{*}=w_{0} \partial \rho_{0} / \partial z^{*}$. Using hydrostatic balance we can also write (4.16) in the form essentially given by Batchelor [2]:

$$
\nabla \cdot \mathbf{v}_{0}-w_{0} \frac{\bar{g}}{c_{s 0}^{2}}=0 .
$$

For the new system $(4.36)-(4.38)$, since $p_{0}$ is a constant when heat conduction is neglected, we have $D \rho_{0} / D t^{*}=0$ and this is why the velocity field is divergence-free. Note that if we seek to "improve" Eq. (4.39) by replacing $\rho_{0}$ by $\check{\rho}=\tilde{\rho}^{*}\left(p_{0}+M^{2} p_{1}, s_{0}\right)$, then we recover the original fully compressible system without simplification! Indeed, the essential difference between the fully compressible system (3.1)-(3.3) (neglecting heat conduction and viscous power terms), and system (4.36)-(4.38) with $p^{*}=p_{0}+M^{2} p_{1}$, is 
precisely that in the new system a term proportional to $M^{2} D p_{1} / D t^{*}$ is neglected in the continuity equation.

This point suggests a modification to the system (4.36)-(4.38) in a situation with possible relevance for atmospheric circulations. Suppose gravity is rather strong but the pressure correction $p_{1}$ does not happen to deviate significantly (more than $O(1)$ ) from some time-independent reference state $\bar{p}\left(z^{*}\right)$ that determines a reference density profile $\bar{\rho}\left(z^{*}\right)$ via a hydrostatic balance equation

$$
\nabla \bar{p}=-\bar{\rho}\left(z^{*}\right) \mathbf{g}^{*} .
$$

Then we replace $\rho_{0}$ in $(4.39)$ by $\bar{\rho}_{0}=\tilde{\rho}^{*}\left(p_{0}+M^{2} \bar{p}, s_{0}\right)$. Note that $\bar{\rho}_{0}$ can depend on $\left(x^{*}, y^{*}, t^{*}\right)$ as well as $z^{*}$ through $s_{0}$. Since $M^{2} D \bar{p} / D t^{*}=M^{2} w_{0}\left(d \bar{p} / d z^{*}\right)$, the continuity equation becomes

$$
\nabla \cdot \mathbf{v}_{0}-w_{0} \frac{\bar{g}}{\bar{c}_{s()}^{2}} \frac{\bar{\rho}}{\bar{\rho}_{0}}=0
$$

where the coefficient $\bar{c}_{s()}^{2}$ is evaluated at $\left(p_{0}+M^{2} \bar{p}, s_{0}\right)$ and we have used $M^{2} g^{*}=\bar{g}$. This equation replaces (4.37), without changing the formula validity of the approximation.

Acknowledgments. This work was partially supported by the National Science Foundation under grants DMS 94-03871 and DMS 97-04924. The second author also acknowledges the support of Krispin Technologies under NASA SBIR contract NAS397087. We thank H. Boukari and R. Gammon for discussions related to this work, and thank R. McLaughlin for recommending reference [23].

\section{REFERENCES}

[1] D. M. Anderson, G. B. McFadden, and A. A. Whecler, Diffuse-interface methods in fluid mechanics, Ann. Rev. Fluid Mech. 30, 139-165 (1998)

[2] G. K. Batchelor, The conditions for dynamical similarity of motions of a frictionless perfect-gas atmosphere, Quart. J. Roy. Meteo. Soc. 79, 224235 (1953)

[3] R. F. Berg, Thermal equilibration near the critical point: Effects due to three dimensions and gravity, Phys. Rev. E 48, 1799-1805 (1993)

[4] H. Boukari, M. E. Briggs, J. N. Shaumeyer, and R. W. Gammon, Critical speeding up observed, Phys. Rev. Lett. 65, 2654-2657 (1990)

[5] H. Boukari, R. Pego, and R. W. Gammon, Calculation of the dynamics of gravity-induced density profiles near a liquid-vapor critical point, Phys. Rev. E 52, 1614-1625 (1995)

[6] H. Boukari, J. N. Shaumeyer, M. E. Briggs, and R. W. Gammon, Critical speeding up in pure fluids, Phys. Rev. A 41, 2260 2263 (1990)

[7] A. J. Chorin and J. E. Marsden, A Mathematical Introduction to Fluid Mechanics, 3rd. ed., SpringerVerlag, New York, 1993

[8] D. L. Denny and R. L. Pego, Solutions for a model of low-speed flow for highly compressible fluids with capillary effects, in preparation

[9] J. E. Dunn and J. Serrin, On the thermomechanics of interstitial working, Arch. Rational Mech. Anal. 88, 95-133 (1985)

[10] D. Durran, Improving the anelastic approximation, J. Atmos. Sci. 46, 1453-1461 (1989)

[11] J. A. Dutton and G. H. Fichtl, Approximate equations of motion for gases and liquids, J. Atmos. Sci. 26, 241-254 (1969)

[12] P. Embid, Well-posedness of the Nonlinear Equations for Zero Mach Number Combustion, Ph. D. Thesis, University of California, Berkeley, 1984

[13] R. Gammon, personal communication. Also see the ZENO home page at the URL http://roissy.umd.edu/. The experimental design is described at the URL http://roissy. umd.edu/usmp3/reminder.html. 
[14] C. Ikier, H. Klein, and D. Woermann, Optical observation of the gas/liquid phase transition in near-critical $S F_{6}$ under reduced gravity, J. Coll. Internat. Sci. 178, 368-370 (1996)

[15] C. Ikier, H. Klein, and D. Woermann, Density equilibration in a near-critical fluid under reduced gravity, Ber. Bunsenges. Phys. Chem. 100 (8), 1308-1311 (1996)

[16] S. Klainerman and A. Majda, Singular limits of quasilinear hyperbolic systems with large parameters and the incompressible limit of compressible fluids, Comm. Pure Appl. Math. 34, 481-524 (1981)

[17] A. B. Kogan, F. Zhong, and H. Meyer, Dynamics of density equilibration near the liquid-vapor critical point of $\mathrm{He}-3$, Czechoslovak J. Phys. 46, Suppl. 1, 71--72 (1996)

[18] L. D. Landau and E. M. Lifshitz, Fluid Mechanics, 2nd ed., Pergamon, Oxford, 1987

[19] F. B. Lipps and R. S. Hemler, A scale analysis of deep moist convection and some related numerical calculations, J. Atmos. Sci. 39, 2192-2210 (1982)

[20] A. Majda, Compressible Fluid Flow and Systems of Conservation Laws in Several Space Variables, Applied Mathematical Sciences, Vol. 53, Springer, New York, 1984

[21] A. Majda and J. Sethian, The derivation and numerical solution of the equations for zero Mach number combustion, Combust. Sci. Tech. 42, 185-205 (1985)

[22] M. R. Moldover, J. V. Sengers, R. W. Gammon, and J. R. Hocken, Gravity effects in fluids near the gas-liquid critical point, Rev. Mod. Phys. 51, 79-99 (1979)

[23] Y. Ogura and N. A. Phillips, Scale analysis of deep and shallow convection in the atmosphere, J. Atmos. Sci. 19, 173-179 (1962)

[24] A. Onuki and R. A. Ferrell, Adiabatic heating effect near the gas-liquid critical point, Physica A 164, 245-264 (1990)

[25] A. Onuki, H. Hao, and R. A. Ferrell, Fast adiabatic equilibration in a single-component fluid near the liquid-vapor critical point, Phys. Review A 41, 2256-2259 (1990)

[26] V. A. Rabinovich, Thermophysical Properties of Neon, Argon, Krypton, and Xenon, Hemisphere Publishing Corp., New York, 1988

[27] R. G. Rehm and H. R. Baum, The equations of motion for thermally driven, buoyant flows, J. Res. Natl. Bur. Stand. 83, 297-308 (1973)

[28] J. S. Rowlinson and B. Widom, Molecular Theory of Capillarity, Clarendon Press, Oxford, 1982

[29] J. V. Sengers, Transport properties of fluids near critical points, Internat. J. Thermophysics 6, 203-232 (1985)

[30] J. V. Sengers, R. S. Basu, and J. M. H. Levelt Sengers, Representative equations for the thermodynamic and transport properties of fluids near the gas-liquid critical point, NASA Contractor Report 3424,1981

[31] H. L. Swinney and D. L. Henry, Dynamics of fluids near the critical point: Decay rate of orderparameter fluctuations, Phys. Rev. A 8, 2586-2617 (1973), and references therein

[32] R. Wilhelmson and Y. Ogura, The pressure perturbation and the numerical modeling of a cloud, J. Atmos. Sci. 29, 1295-1307 (1972)

[33] C.-S. Yih, Stratified Flows, Academic Press, New York, 1980

[34] B. Zappoli and P. Carles, The thermo-acoustic nature of the critical speeding up, Eur. J. Mech. B/Fluids 14, 41-65 (1995)

[35] B. Zappoli, S. Amiroudine, P. Carlès, and J. Ouazzani, Thermoacoustic and buoyancy-driven transport in a square side heated cavity filled with a near critical fluid, J. Fluid Mech. 316, 53-72 (1996)

[36] F. Zhong and H. Meyer, Density equilibration near the liquid-vapor critical point of a pure fluid: Single phase, Phys. Rev. E 51 3223-3241 (1995) 